\title{
Weighting National Survey Data: why, how and which weight?
}

\section{Ferdous Hakim}

Mental and Neurology Study Team, National Institute of Mental Health

\section{Rijwan Bhuiyan}

Mental and Neurology Study Team, National Institute of Mental Health

\section{Mst. Khaleda Akter}

Mental and Neurology Study Team, National Institute of Mental Health

\section{Md. Mohit Kamal}

National Institute of Mental Health

\section{Md. Faruq Alam}

National Institute of Mental Health

\section{Md. Rizwanul Karim}

Directorate General of Health Services

Mohammad Mostafa Zaman ( $\sim$ zamanm@who.int )

World Health Organization https://orcid.org/0000-0002-1736-1342

\section{Technical advance}

Keywords: weighting, base weight, adjusted weight, non-response, population calibration, trimming, precision, bias, sample distribution

Posted Date: May 28th, 2020

DOI: https://doi.org/10.21203/rs.3.rs-29633/v1

License: (c) (i) This work is licensed under a Creative Commons Attribution 4.0 International License.

Read Full License 
Version: 19 May 2020

\section{Weighting National Survey Data: why, how and which weight?}

Authors:

Ferdous Hakim¹, Rijwan Bhuiyan², Mst. Khaleda Akter ${ }^{3}$, Md. Mohit Kamal ${ }^{4}$, Md. Faruq Alam5, Md. Rizwanul Karim ${ }^{6}$, M Mostafa Zaman ${ }^{7}$

1 Mental and Neurology Study Team; National Institute of Mental Health; Cell: +880178768799; Email: drferdoushakim@gmail.com; ORCID number: https://orcid.org/0000-0003-2376-3978

2 Mental and Neurology Study Team; National Institute of Mental Health; Cell: +8801701205881; Email: physiorijwan@gmail.com; ORCID number: https://orcid.org/00000003-0005-8889

3 Mental and Neurology Study Team; National Institute of Mental Health; Cell: +8801701202989; Email: khaleda.akter.auw@gmail.com; ORCID number: https://orcid.org/0000-0001-8857-1649

4 Ex-Director and Professor, National Institute of Mental Health; Cell: +8801711832955 ; Email: drmohitkamal@yahoo.com;

5 Ex-Director and Professor, National Institute of Mental Health; Cell: +8801711244749; Email: faruqalam60@gmail.com;

6 Associate Professor and Program Manager Mental Health and Disability, Directorate General Health Services; Cell: +8801711639983; Email: shameem.m25@gmail.com;

7 World Health Organization Bangladesh; Cell: +8801714165205; Email: zamanm@who.int; ORCID number: https://orcid.org/0000-0002-1736-1342

Number of words (except tables and figures): 2947

Number of tables: 4

Number of figures: 2 


\section{Corresponding Author:}

M Mostafa Zaman

Research \& Publication Advisor, and Gender Equity and Rights Focal Point; World Health Organization Bangladesh, United House (GF to 3rd Floor), 10 Gulshan Avenue, Gulshan-1

Dhaka-1212, Bangladesh; PO Box: 250; Telephone: 8809604027200 (Hunting); Fax: (+88 02) 8831423; Cell: +8801714165205; Email: zamanm@who.int 


\begin{abstract}
Background

Weighting of national data is a procedure that enables the sample to be more representative of the target population. Weighting procedure is a thorough exercise and yields several types of weights. However, considerable variation exists among authors on which weight to use leaving the researchers baffled. In this article we share our experience on weighting for a few recent national surveys in Bangladesh.
\end{abstract}

\title{
Methods
}

We generated four weights: the base weight calculated from probabilities of selection, and nonresponse adjustments, population calibration, and trimmed weights. Finally we checked weighted means, medians, ranges, standard errors, confidence intervals, variances, multiplicative effects, design effects and prevalence of a key variable of the survey to decide on which weight to use.

\section{Results}

Compared to unweighted distribution, weighting makes the sample distribution to conform to the population. Among the four calculated weights, the trimmed weight had narrow standard error and variance, and smallest design and multiplicative effects. It yielded an acceptable prevalence and distribution of a core variable.

\section{Conclusion}

Though weighting is a time intensive exercise, it had a favorable effect on the sample distribution to comply with the Bangladeshi population. Among the four weights, we show that the trimmed weight met all parameters of good quality and precision. Therefore, we recommend to use this weight for national level surveys in Bangladesh.

Word count: 217 


\section{Key words}

weighting, base weight, adjusted weight, non-response, population calibration, trimming, precision, bias, sample distribution 


\section{Background}

Sample survey is one of the most important methods of collecting health data that can draw conclusion on a reference population(1). However inference cannot be drawn without treating the sample data. Weighting corrects the imperfections in the sample that prevents bias and other departures between the sample and the reference population. In complex sample surveys four types of imperfections emerge from unequal probabilities of selections, multistage selection, stratifying sample into the reporting domains, and non-responses and population noncoverage(2). Ignoring these will lead to incorrect inferences in a survey.

Though sample survey can draw conclusion to a reference population(1), the results may be influenced by sampling and non-sampling errors(3). Among the non-sampling errors, nonresponse - both unit non-response and item non-response - is addressed rigorously through weighting. Adjustment of the non-sampling error can be different depending on the data collection technique of the survey - digital and paper-pencil. Digital data collection has a wellordered method to adjust non-sampling error compared to pen-paper based one(4). Several recent national level household $(\mathrm{HH})$ surveys in Bangladesh(5)(6)(7) used digital data collection tools.

In addition, weighting adjusts the weighted sample distribution for key variables of interest (for example, age, race, and sex) to make it conform to a known population distribution(8).

Production of design-unbiased estimates of parameters of interest is possible by applying proper weights(9). Thus weighting procedure is a critical step after the survey data have been collected and all the essential steps of data processing have been completed(10).

Testing the variability of the calculated non-response rates and weight is an important step of generating acceptable weights(5). High variation in weights can lead to some observations having too much importance. These might lead to distortion of survey results. In addition if the sampling design is not informative, using the weights or not should not introduce any significant 
differences in the estimates and if the sampling design turns out to be informative, the use of weighted estimators will produce "better" results(9).

Weighting itself to some researchers is like a black box(10). Handling different types of weights and which to use sometimes lead researchers confused. As a result survey data are often used by researchers without the weights leading to erroneous conclusions(9). Given the influence weights have on survey results, it is important that researchers understand enough about weighting process to be discerning users of the survey data(10). In this article we describe the weighting process, our approach in identifying which weight to use and explain the reason behind selecting one. We believe that there is no scientific article on weighting of survey data by authors from Bangladesh and our article is the first of its kind in the context of health survey data in Bangladesh.

\section{Methods}

\section{A. Overview of the sampling and weighting procedure}

Several recent national level household $(\mathrm{HH})$ surveys conducted in Bangladesh - having similar design (geographically clustered, multistage, probability based)(5)(6)(7) and frame(11) - used digital data collection tools. The results shown in this article are extracted from the National Mental Health Survey Bangladesh 2019 which was ethically approved by the Bangladesh Medical and Research Council.

The estimated sample size was 8928 selected in three stages. The first stage was to randomly select primary sampling units (PSUs) from the frame of PSUs covering $95.85 \%$ of the total population of Bangladesh(11). The PSUs (496) were equally selected from each of the eight divisions segregated into urban and rural strata. From each of the selected PSUs the required and equal number (18) of HHs were selected by systematic sampling - the second stage. These $\mathrm{HHs}$ were equally allocated to either of man or woman $\mathrm{HH}$ (sex randomization). One individual 
was randomly selected from a list of eligible member of each $\mathrm{HH}$ in the third stage (Figure 1). The overall response rate of the survey was $90.4 \%(12)$ (Table 1).

There is no universally held protocol for calculating weights(5). The aim of the weighting procedure is to calculate the 'final weight'. We used Microsoft Excel in Microsoft Office 365 bundle for this exercise. The 'base weight' calculation was the initial step. The adjustment of the base weights for non-response was the second step. The third step was to adjust the weights to a known population total. In the fourth stage a trimming exercise was applied.

\section{Base weight calculation:}

Base weight can be calculated soon after sampling exercise and $\mathrm{HH}$ listing activity. It involves the probability of selection of PSUs (p1), households (p2), sex randomization (p3) and individuals (p4). Probability is the respondent's original selection probability based on the sample design(6)

\section{(Figure 1).}

i. PSU selection probability ( $p 1)$ : Selection for PSUs within each of the 16 strata (8 division $\mathrm{x}$ 2 residence status) is done.

$$
p 1=\frac{\text { Number of selected PSUs in a strata (31) }}{\text { Total number of PSUs in that strata }}
$$

ii. HH selection probability (p2): It is calculated by systematic selection of $18 \mathrm{HHs}$ within each PSU. It is performed in 496 PSUs.

$$
p 2=\frac{\text { Number of selected HHs in a PSU (18) }}{\text { Total number of HHs in that PSU }}
$$

iii. Probability of sex randomization (p3): Equal household sex allocation of the selected 18 $\mathrm{HHs}$ are undertaken in each PSU. This yielded a probability of ' 0.5 '. This is applicable for all (8 928) the respondents of the survey.

$$
p 3=\frac{\text { Number of selected sexes (1) }}{\text { Total number of sex strata (2) }}
$$


iv. Individual selection probability (p4): The selection probability for one eligible individual among a number/s of eligible $\mathrm{HH}$ members is calculated. This is obtained from the survey response data and is applicable to all the respondents of the survey.

$$
p 4=\frac{\text { Number of selected } H H \text { memebr }(1)}{\text { Total eligible } H H \frac{\text { member }}{S}}
$$

The base weight is calculated as,

$$
\text { base weight }=\frac{1}{p 1} \times \frac{1}{p 2} \times \frac{1}{p 3} \times \frac{1}{p 4}
$$

\section{Non-response weight:}

In this step, we need to estimate the probability of responses/ non-responses using information available for both respondents/ non-respondents. These are generated as 'disposition codes' (Table 1) in a survey dataset.

The non-response weight is calculated by taking the inverse of the response rate for each subset of the survey. The non-response adjustment is undertaken at PSU, $\mathrm{HH}$ and individual levels.

i. PSU-level non-response factor: This is applied for 16 strata (8 division $\times 2$ residence status). It is calculated as,

$$
\text { PSU non }- \text { response }=\frac{\text { Sum of base weight of eligible PSUS }}{\text { Sum of base weight of completed PSUS }}
$$

ii. Household non-response factor: It is calculated within each PSU, so there are 496 adjustment cells. The $\mathrm{HH}$ level non-response adjustment is calculated as,

$$
H H \text { non }- \text { response }=\frac{\text { Sum of base weight of eligible households }}{\text { Sum of base weight of completed households }}
$$

iii. Person non-response factor: It is calculated by gender (e.g. 2 groups), age group (e.g. four groups) and a core variable of interest (e.g. two groups) of the survey in concern.

Therefore, the person non-response is calculated in $16(2 \times 4 \times 2)$ adjustment cells. The person-level non-response adjustment is,

Individual non - response $=\frac{\text { Sum of base weight of eligible households }}{\text { Sum of base weight of completed rosters }}$ 
The non-response adjusted base weight is calculated by multiplying the three non-response factors with the base weights successively.

$$
\begin{aligned}
& \text { Non - response adjusted base weight } \\
& \qquad=\text { base weight } * \text { non-reposnses weights (PSU x HH x Individual) }
\end{aligned}
$$

\section{Population weight/ Calibration:}

If census information is available these can be used for the correction of over- or underrepresentation in the sample of the targeted age-sex groups. In principle, the goal of a calibration weight adjustment is to bring weighted sums of the sample data in line with the corresponding counts(13) and frame deficiencies(14) in the target population.

Initially projected population is estimated then population calibration factor is calculated.

\section{Estimating the projected population from census data}

The steps of population estimation are as follows:

1. Summarizing the population by domains of residence ( 2 strata), sex ( 2 strata), and age groups (5 strata) for each division (8 strata)(11);

2. Estimating the total projected population from census month up to the data collection month is done using exponential growth rate for each of the strata.

\section{Calculating post stratification adjustment factor (r)}

The population calibration factor is calculated by division, residence, gender and the five age groups resulting in $160(8 \times 2 \times 2 \times 5)$ adjustment cells. The post-stratification adjustment is calculated as:

$$
\text { population calibration factor }=\frac{\text { projected population in a domain }}{\text { non response adjusted weights in that domain }}
$$

Population calibrated weights are calculated by multiplying the non-response adjusted base weight with population calibration factor.

\section{Trimming of weight}


The trimming procedure varies between researchers (15) (16). Trimming can be applied to any stage of the weighting process (10). In our exercise we followed a trimming procedure based on the assumed distribution of sampling weights (16). The process started with identifying the extreme weight/s and fixing all weights above and below the set cut-off points at that value. The weights thus lost were equally distributed among the non-trimmed weights. This procedure was repeated till no weights were above the cut-off point (15).

\section{B. Checking the calculated weights}

We thoroughly checked all the steps of calculations for the weighting process. In addition we were keen on checking the distribution of the weights specially taking notice of the extreme values and back-tracking these for possible errors for quality results. We also checked the weights with standard statistical parameters like: mean, median, range, standard error, confidence interval, variance, multiplicative effect, design effect and prevalence of a key variable of the Survey.

\section{Results}

We calculated the four weights using the mathematical formula mentioned in the method section.

\section{1. 'Base weight' calculation:}

Probabilities of selection of PSUs, HHs, sex randomization and individual selection probability were taking into consideration. This is applied to 16 strata comprised of eight divisions and two residence strata. This procedure yielded a total base weight value of 79 422 102. (Table 2)

\section{Non-response factor calculation:}

i. PSU-level non-response factor: This is also calculated for 16 domains. The value for the PSU non-response is essentially ' 1 ' for all the 15 domains except in the one domain where on PSU was dropped. The mean PSU non-response is 1.0008 . 
ii. Household non-response factor: This is calculated in all 496 PSUs. The mean PSU nonresponse is 1.1002 .

iii. Person non-response factor: The mean PSU non-response is 1.1002.

When this base weight is adjusted with the non-response weights, the adjusted base weight stands at 92569 866. (Table 2)

\section{Calculating the projected population from census data:}

Data is extracted from Population and Housing Census 2011(11) disaggregated by sex and five groups of age in years for each of the eight divisions of Bangladesh. The growth rate published by the census report is used and a duration of eight years is applied to calculate the projected population from February 2011 up to March 2019.

The total projected population calculated for adults aged 18 years or more is: 102161911 . However when population and housing census was conducted in 2011, Bangladesh was divided into 7 administrative divisions. Later on a new Division, Mymensingh was created. So we extracted the required population counts of the districts included under this Division from the

Dhaka division. (Table 2)

\section{Population weight/ Calibration (r):}

This is calculated in 160 domains: 8 divisions, 2 residence strata, 2 sex strata and 5 age groups. In each of the domains the sum of projected population in that domain is divided by the nonresponse adjusted base weights of that domain. The mean ' $r$ ' was 1.328213. (Table 2)

\section{Calculating population calibration and non-response adjusted weight:}

This weight is the product of base weight; PSU, household, and individual non-response factors; and population calibration factor. Our calculated adjusted weight was 102948678.

\section{(Table 2)}

\section{Trimming of weight:}

In our exercise we trimmed the non-response and population calibration adjusted base weight. We identified the median of the non-response adjusted and population calibrated weight to be 9 
091.9. All weights above and below the 3.5 times median(15)(16) value of 31821.7 and was set at that value. We trimmed any weight above 31821.7 and fixed the weight at that value. (Table 3)

\section{Testing the calculated weights}

First a comparison was made between the distribution of the projected population with the unweighted sample to show the differences in distribution by age and residence. (Figure 2A and B). It is shown that the unweighted sample distribution is not similar to the population distribution. However when we make the same comparison with weighted distributions with any of the four calculated weights, it shows that the distribution closely matches with that of population. The best match was achieved by the sample distribution weighted with population calibrated and trimmed weights. (Figure 2)

All the weights except the trimmed weight show a wide range denoting instability of the calculated weights. Sum of the calculated weights gradually increased from the base weight to the trimmed weights. The population calibrated weights and the trimmed weights thus stands at $100.8 \%$ (Table 3 ) of the projected population. The distribution of the trimmed weight is more centrally oriented as is denoted by the difference between maximum and minimum, narrower standard error, confidence level than other weights. The multiplicative effect for the trimmed weight is 1.5 and the only weight which is less than 2 .

We also checked the effect on the different weights on the prevalence of mental disorders according to the National Mental Health Survey $2019(7)$. The unweighted prevalence is $17.3 \%$. Which is very close to the weighted prevalence $(15.8 \%-16.8 \%)$. We also calculated the prevalence in urban-rural and male and female domains and found no notable difference. However, overall the prevalence of mental disorders tend to decrease as we progress from unweighted to weighted results. However, we think that this difference is negligible. We calculated the design effect of unweighted and weighted calculations. Though it is somewhat increased in the weighted results, we observe the lowest design effect for base weights (1.7) and trimmed weights (1.7). (Table 4) 


\section{Discussion}

We calculated four weights: base, non-response adjusted, population calibrated and trimmed weights using Microsoft Excel. As population counts were not available for the Survey year (2019) for Bangladesh, we estimated projected population from the 2011 census(11). In addition we had to extract population data one division as the number of divisions after 2011 census increased from seven to eight. We provide evidence that weighting yields a favorable result compared to the unweighted inferences. In addition, the weighting adjustments add more precision maintaining validity of results. Weighting of national surveys requires keen knowledge on survey sampling procedure and non-responses. Though the process in theory is quite straightforward, the calculation is not. Considerable variation exists among authors whether to adjust or not adjust, how to form weighting class and post-stratification cells, whether and how to trim the weights, and so forth(10). We presented here the weighting process from a recently conducted National Mental Health Survey 2019(7) and tested those for quality(5).

It is claimed that weighting with base weight only is an efficient method as it is a simple one to construct(10). It be completed after the mapping and listing activity before the data collection. It avoids the performing meticulous non-response calculations and the need for population projection estimation and calibration. Thus base weight can be used as the final weight for a survey when response rate is $90 \%$ or more(17). Otherwise calculating a non-response and population calibration adjusted base weight is recommended(5). However we generated all four weights despite survey response rate was acceptable and fresh census data unavailable.

In the recent surveys as data were collected though handheld computers item non-response was completely absent(4). However the weighting procedure corrected the sample distribution for unit non-response. The compared to the unweighted distribution of sample, the weighted distribution were more reflective of population distribution and size. In contrast the unweighted sample distribution did not meet this requirement.

Despite the sampling design with equal allocation of PSUs to urban-rural and male-female strata, the calculated weights corrected the sample distribution for variables like sex, residence etc. to 
make it conform to the population by distribution and size(8). This achieves one of the prime objectives of the weighting exercise(15). The biasness induced by the design effect is also reduced by the small design effect in the weighted results. Small design effect will help to estimate a smaller required sample size which is much needed in a low-resource country. In addition we calculated trimmed weights(15). There are issues both in favor and against trimming of the weights(17)(15). Some authors do not recommend this procedure as it might induce inaccurate results(18). Trimming survey weights introduces a small bias into estimates but greatly reduces standard errors(17). The cost of weighting data is reduced accuracy/ precision to some extent. Some survey practitioners worry about dealing with highly unequal weights which trimming might address thus improving precision(15). We encountered this situation in the generated weights. In our study the trimmed weight was stable and provided a favorable result.

We tested(10)(5) these weights for compensation of non-sampling errors, matched with population distribution, variability and accuracy. It has been argued that even if weights reduce bias, they might largely inflate variance of estimates(19). Though we encountered a little loss of precision overall in the process if base weight is used, this is gradually removed when we use the other weights. The results calculated using the trimmed weight having the most precision. Except for the trimmed weight calculated weights showed a large range of values denoting instability of the weights. In our data we showed that trimming procedure generated a weight that stroke a good balance between instability and accuracy.

\section{Conclusion}

Weighting compensated for the non-sampling errors and corrected the imperfections in the sample and prevented bias between the sample and the reference population in contrast to the unweighted sample. We found that the trimmed weight was the most acceptable among the four weights. The results generated by using the trimmed weights yields a more nationally representative, precise results and renders it comparable with other national data. 


\section{List of abbreviations}

\begin{tabular}{ll}
\hline Abbreviation & Expanded form \\
\hline PSU & Primary sampling unit \\
$\mathrm{HH}$ & Household \\
\hline
\end{tabular}

\section{Declarations}

\section{Ethics approval and consent to participate}

All the respondents provided informed written consent to participate in the study. The study was approved by Bangladesh Medical and Research Council (Reference No.

BMRC/NREC/2016-2019/80).

\section{Consent for publication}

Not applicable.

\section{Availability of data and materials}

The datasets used and/or analysed during the current study are available from the corresponding author on reasonable request.

\section{Competing interests}

The authors declare that they have no competing interests.

\section{Funding}

No fund was required to undertake the exercise described in the manuscript.

\section{Authors' contribution}

FH computed the weights, analyzed the data, drafted and refined the manuscript after contribution by the co-authors. MRB participated in computing the weights, contributed to the draft manuscript and collected reference papers. MKA provided support in population projection and provided inputs to the draft manuscript. MMK, MFA and MRK provided comments in the draft manuscript. and MMZ guided the weighting process, conceptualized the 
theme, and reviewed the data and manuscript critically. All authors approved the final version of the manuscript to be submitted for publication.

\section{Acknowledgements}

The authors gratefully acknowledge support of Mr Tahidul Islam, Deputy Director, Bangladesh Bureau of Statistics, for population projection based on Housing and Population Census of Bangladesh 2011. Unpublished works of done by National Institute of Mental Health - used in this report - have been done by investigators beyond the author list.

\section{Disclaimer}

None declared. The authors alone are responsible for the views expressed in this article and they do not necessarily represent the views, decisions or policies of the institutions with which they are affiliated. 


\section{References}

1. Cochran WG. Sampling techniques. Wiley; 1977.428 p.

2. Bell BA, Onwuegbuzie AJ, Ferron JM, Jiao QG, Hibbard ST, Kromrey JD. Use of design effects and sample weights in complex health survey data: A review of published articles using data from 3 commonly used adolescent health surveys. Vol. 102, American Journal of Public Health. American Public Health Association; 2012. p. 1399-405.

3. Butar Butar F, Chang C. Weighting Methods in Survey Sampling Permutation method View project Weighting Methods in Survey Sampling [Internet]. 2012. Available from: https://www.researchgate.net/publication/313036499

4. Lohr SL, Grove P, Belmont A. Sampling: Design and Analysis QP $\AA^{8}$ An International Thomson Publishing Company

5. Global Adult Tobacco Survey Collaborative Group. Global Adult Tobacco Survey (GATS) GTSS Sample Weights Manual [Internet]. Altanta; 2010 [cited 2020 Jan 25]. Available from:

https://www.who.int/tobacco/surveillance/9_GATS_SampleWeightsManual_v2_FINAL_15 Dec2010.pdf

6. World Health Organization. WHO STEPS Surveillance Manual. Geneva; 2017. 472 p.

7. National Institute of Mental Helath. National Mental Health Survey Bangladesh 2019 Provisional Fact Sheet (to be revised) [Internet]. Dhaka: NIMH; 2019. Available from: https://dghs.gov.bd/images/docs/Notice/NIMH Fact Sheet final-5-11-19.pdf

8. Yansaneh IS. Household Sample Surveys in Developing and Transition Countries Chapter II Overview of sample design issues for household surveys in developing and transition countries.

9. Lavallee P, Beaumont J-F. Why We Should Put Some Weight on Weights. Surv Methods Insights from F. 2015;1-18. 
10. Leeuw EDD, Hox J DD. International Handbook of Survey Methodology [Internet]. 1st ed. New York: Taylor and Francis; 2008 [cited 2020 Mar 12]. 560 p. Available from:

https://books.google.com.bd/books?id=19mVYNUvbpgC\&pg=PA339\&lpg=PA339\&dq=rese archers+are+in+confusion+on+weighting+of+survey+data\&source=bl\&ots=w5Dn6fFaWx\& sig=ACfU3U39Zs_ScgMKLrjE-

WFFF5ujAf6PZg\&hl=en\&sa=X\&ved=2ahUKEwjOiOHgsJToAhWf4jgGHYfNA04Q6AEwC3 oECAoQAQ

11. Bangladesh Bureau of Statistics. Population-and-Housing-Census 2011 [Internet]. 2011 [cited 2020 Jan 25]. Available from: http://www.bbs.gov.bd/site/page/47856ad0-7e1c4aab-bd78-892733bc06eb/Population-and-Housing-Census

12. National Institute of Mental Helath. National Mental Health Survey Bangladesh 2019 Provisional Fact Sheet [Internet]. Dhaka; 2019. Available from: https://www.who.int/docs/default-source/searo/bangladesh/pdf-reports/cat-2/nimh-factsheet-5-11-19.pdf?sfvrsn=3e62d4b0_2

13. Johnson DR. Using Weights in the Analysis of Survey Data [Internet]. Power point presentation. 2008 [cited 2020 Jan 25]. Available from:

http://www.nyu.edu/classes/jackson/design.of.social.research/Readings/Johnson Introduction to survey weights \%28PRI version\%29.pdf

14. Reig J. (Very) basic steps to weight a survey sample [Internet]. 2017 [cited 2020 Jan 25]. Available from: https://bookdown.org/jespasareig/Book_How_to_weight_a_survey/

15. Valliant R, Dever JA, Kreuter F. Practical tools for designing and weighting survey samples [Internet]. Pittzburg: Springer; 2013 [cited 2020 Jan 25]. 670 p. Available from: https://www.springer.com/gp/book/9781489993816

16. Potter F, Zheng Y. Methods and Issues in Trimming Extreme Weights in Sample Surveys [Internet]. [cited 2020 May 3]. Available from: https://nces.ed.gov/nationsreportcard/tdw/weighting/2008/ltt_weighting_2008_trimming_ad 
ju

17. Mohadjer L. Sample Weights for Households with Multiphase Data Collection Approaches [Internet]. Maryland; 1999 [cited 2020 Jan 25]. Available from:

https://www.stat.fi/isi99/proceedings/arkisto/varasto/moha0259.pdf

18. D. R. Johnson. Using weights in the analysis of survey data [Internet]. 2008 [cited 2020 Jan 25]. Available from: http://www.wisc.edu/

19. Organisation for Economic Co-operation and Development. Survey weighting and the calculation of sampling variance [Internet]. Paris; 2015 [cited 2020 May 4]. Available from: https://www.oecd.org/pisa/sitedocument/PISA-2015-Technical-Report-Chapter-8-SurveyWeighting.pdf 


\section{Figure titles}

Figure 1. Sampling stages and probabilities of selection of a Survey(12)

Figure 2. Comparison of population* $(A)$ with unweighted $(B)$ distributions and weighted sample distributions C (base weight), D (non-response adjusted), E (population calibrated), and F (trimmed) in percent

* Projected population of Bangladesh aged $\geq 18$ years is based on Census 2011 (11) 


\section{Figures}

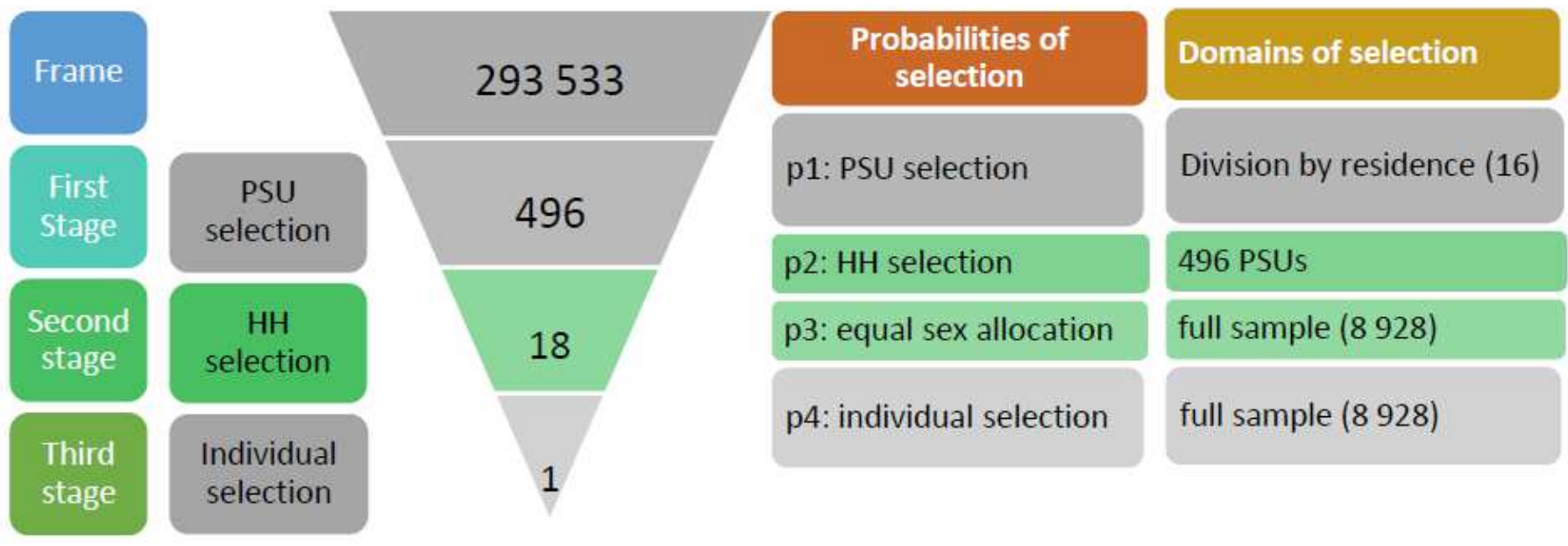

\section{Figure 1}

Sampling stages and probabilities of selection of a Survey(12)

A.

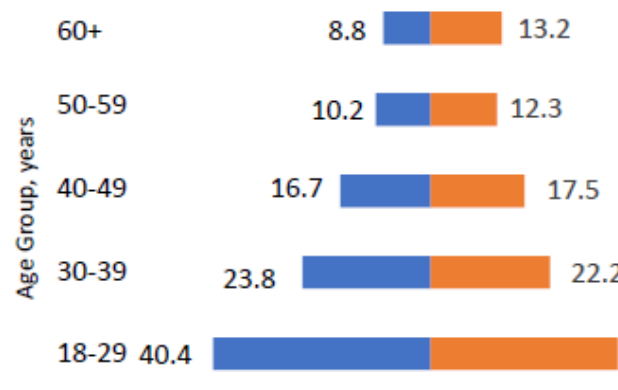

C.
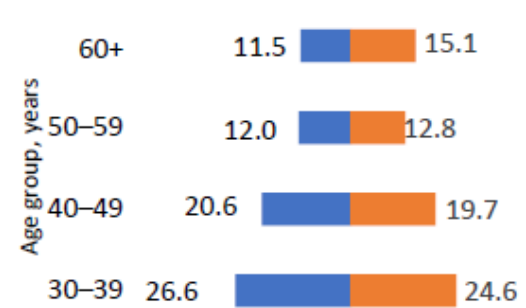

18-29 29.3

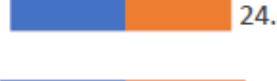

$27.9 \quad 29.3$

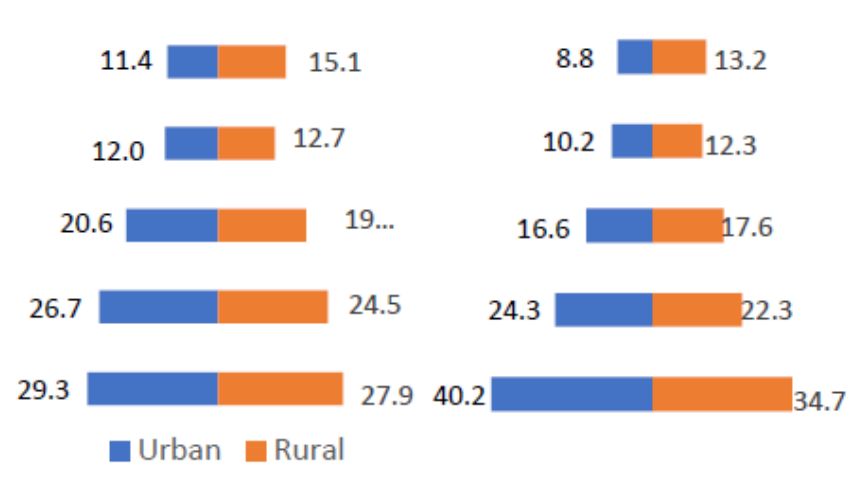

B.

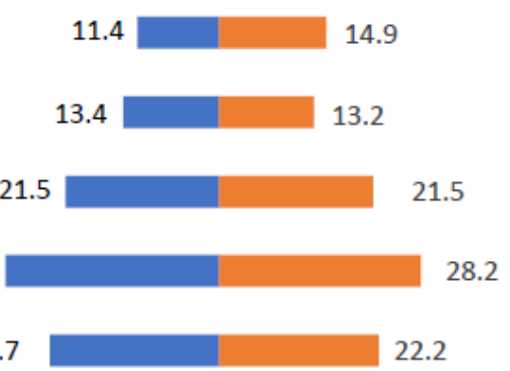

E.

F.

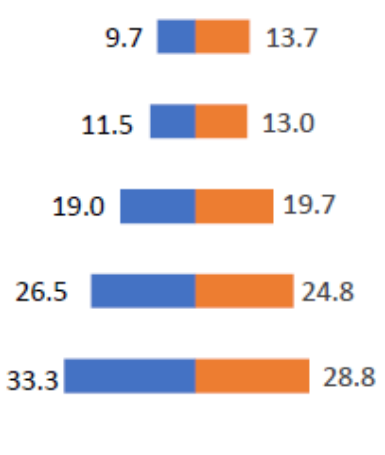

\section{Figure 2}

Comparison of population* (A) with unweighted (B) distributions and weighted sample distributions C (base weight), D (non-response adjusted), E (population calibrated), and $F$ (trimmed) in percent * 
Projected population of Bangladesh aged $\geq 18$ years is based on Census 2011 (11)

\section{Supplementary Files}

This is a list of supplementary files associated with this preprint. Click to download.

- WeightingNationalSurveyDatatableshakimf17May2020.pdf 